\title{
Treatment of Granulomatous Mastitis: Is There a Role for Antibiotics?
}

\author{
(1) Meagan S. Williams ${ }^{1}$, (1) Adelaide H. McClintock ${ }^{1}$, (1) Lori Bourassa ${ }^{2}$, (D) Mary B. Laya ${ }^{3}$ \\ ${ }^{1}$ Department of Medicine, Division of General Internal Medicine, University of Washington, Washington, USA \\ ${ }^{2}$ Department of Laboratory Medicine and Pathology, Division of Clinical Microbiology, University of Washington, Washington, USA \\ ${ }^{3}$ Department of Medicine, Division of General Internal Medicine, Breast Care Program, University of Washington, Washington, USA
}

\begin{abstract}
Objective: To perform a retrospective review of the clinical characteristics, microbiological data, and clinical outcomes in patients with granulomatous mastitis (GM) who were treated at our institution with a unique strategy of prolonged antibiotic therapy as the primary treatment modality.

Materials and Methods: A retrospective case series was performed on patients $(n=42)$ with GM seen at the breast specialty clinic of our institution between the years 2004 and 2014. Patients were primarily treated with lipophilic antibiotics, and steroids and surgery were reserved for refractory cases.

Results: Bacteria were identified in 34 samples from 22/42 patients (52.3\%). Diphtheroids (presumptive Corynebacterium spp.) were most commonly identified, followed by Corynebacterium spp. and Propionibacterium acnes (now Cutibacterium acnes). Antibiotics were our preferred first-line medical therapy and were used in 33/36 (91.7\%) patients. The mean duration of antibiotic therapy was $7.0 \pm 4.5$ months. Clarithromycin was our antibiotic of choice and was the initial antibiotic used in 15 of the 33 patients (45.5\%) treated with antibiotics. Eleven patients required adjunctive therapy with prednisone. The mean duration of steroid therapy was $4.3 \pm 2.5$ months. Surgery for therapeutic purposes included incision and drainage in seven patients, fine needle aspiration in eight patients, and excision of the fistulous tract in one patient. No patients had large-volume excisions. The average time from the first breast clinic visit to clinical resolution was $8.0 \pm 4.6$ months.

Conclusion: GM may be the result of a bacterial process that induces a unique form of inflammatory response. Clinicians should consider special requests to microbiology laboratories to attempt to isolate Corynebacterium spp. in the evaluation of samples sent to the laboratory for analysis. An extended course of a lipophilic antibiotic is a largely unexplored but potentially effective treatment option with low associated morbidity. More research is needed in this area.

Keywords: Granulomatous mastitis, idiopathic granulomatous mastitis, breast disease, benign breast disease
\end{abstract}

Cite this article as: Williams MS, McClintock AH, Bourassa L, Laya MB. Treatment of Granulomatous Mastitis: Is There a Role for Antibiotics?. Eur J Breast Health 2021; 17(3): 239-246

\section{Key Points}

- A retrospective case series was performed on patients with GM seen at the breast specialty clinic of our institution.

- Diphtheroids (presumptive Corynebacterium spp.) were the most commonly identified bacteria in breast tissue samples, followed by Corynebacterium spp. and Propionibacterium acnes (now Cutibacterium acnes)

- $\quad$ Patients were primarily treated with lipophilic antibiotics; steroids and surgery were reserved for refractory cases.

- The mean duration of antibiotic therapy was $7.0 \pm 4.5$ months, and the mean duration of steroid therapy was $4.3 \pm 2.5$ months. The average time from the first breast clinic visit to clinical resolution was $8.0 \pm 4.6$ months.

- Clinicians should consider requesting inclusion of Corynebacterium spp. in the evaluation of samples sent for laboratory analysis by asking for specieslevel identification of any corynebacteria recovered in culture, adding Tween 80 (a lipid source) and esculin to growth media, and incubating cultures for a sufficient duration to recover slow-growing organisms such as C. kroppenstedtii.

- An extended course of a lipophilic antibiotic, such as clarithromycin, that has adequate tissue penetrance within lipid-filled spaces may be an option for treating GM. 


\section{Introduction}

Granulomatous mastitis (GM) is a challenging clinical condition first described in 1972 by Kessler and Wolloch (1). There remains a lack of consensus around both a unifying pathophysiologic model of this disease and a standard treatment protocol. Patients often present to a women's health or primary care provider and are frequently initially managed as typical non-lactation mastitis or malignancy (2). After failure of a standard short course of antibiotics, chronic inflammation develops, often with the formation of a fistula and an abscess (2-4).

Multiple theories have been proposed about the etiology of GM, including autoimmune disease, elevated hormonal states such as hyperprolactinemia, and infection (3). However, none of these theories have been widely accepted, hence the continued reference to this disease as "idiopathic" GM in the literature. There exists wide variability in treatment approaches, including observation only $(5,6)$, short courses of antibiotics, long courses of immunosuppressants (2, 7-9), and surgical interventions (10).

Common immunosuppressant therapies include methotrexate and corticosteroids $(2,8,11)$. There is a pathophysiologic basis for corticosteroid use, since steroids inhibit granuloma formation through inhibition of the production of inflammatory cytokines (TNF alpha and IFN-gamma) and are used in other noninfectious granulomatous diseases such as sarcoidosis (12). Surgical approaches vary depending on clinical presentation and can include incision and drainage, wide local excision, or even mastectomy $(10,13,14)$. Differing degrees of success have been reported in the literature with regard to surgery, with some studies concluding that surgery is the ideal treatment strategy (15-17) and others either advising against it or reserving it for refractory cases only due to surgical site complications, recurrence after excision, and morbidity associated with large-volume excisions $(14,18,19)$.

In 2003, Taylor et al. (20) were the first to observe the presence of Gram-positive bacilli within granulomas of women with GM and also noted that Corynebacterium kroppenstedii was isolated in $44 \%$ of their case series. This led to the assertion that corynebacteria, though often considered to be contaminants, are likely truly pathogenic when isolated from breast tissue. Subsequently, several other studies have demonstrated an association of GM with Corynebacterium spp. (21-24). However, consistent isolation remains a challenge as some Corynebacterium spp. require prolonged incubation or specialized growth media. C. kroppenstedii in particular requires lipid supplementation to grow consistently on synthetic media (25). This lipid requirement may explain its propensity for growth in lipidrich environments, such as that found in mammary glands. Even if Corynebacterium spp. do grow on conventional media, they may be dismissed as non-pathogenic "diphtheroid" skin contaminants. Dobinson also noted that isolation of the bacteria declines on subsequent cultures (23).

Prior studies with short courses of beta lactams have demonstrated low rates of resolution. However, this may be attributable to the high minimum inhibitory concentrations of these antibiotics in Corynebacterium spp. $(23,24,26)$. Additionally, the bacteria
Granulomas are highly complex immune system structures formed in response to a variety of infectious and noninfectious agents. In the quintessential granulomatous infection, infection with Mycobacterium tuberculosis, granulomas appear to be an only partially effective host defense which, though containing the bacilli, may also provide safe shelter from the immune system (27, 28). This may in part explain the necessity of prolonged courses of antibiotics, with treatment needed for many months to years before clinical resolution.

More recently, longer courses of lipophilic antibiotics have been proposed, to allow for better penetration and tissue concentration of antibiotics $(23,29)$, as Corynebacterium spp. are typically found within lipid-filled vacuoles within the granuloma rather than in the surrounding inflamed tissue.

Given the growing body of literature suggesting an infectious etiology for GM, particularly by corynebacteria, we developed an approach to evaluation and treatment that was targeted toward an infectious process with these organisms in particular. This strategy involve 1) requesting species-level identification of Corynebacterium spp. isolated in culture or identified by polymerase chain reaction (PCR), 2) the use of lipophilic antibiotics or higher doses of non-lipophilic antibiotics for tissue penetrance, 3) prolonged medical treatment, since the structure and function of granulomas inhibit antibiotic effectiveness, 4) adjuvant glucocorticoid treatment for refractory cases, and 5) surgery when drainable lesions were present or for symptomatic treatment if desired. The purpose of this study was to review the clinical characteristics, microbiological data, and clinical outcomes of patients managed with this approach.

\section{Materials and Methods}

A retrospective chart review was performed on patients with GM seen at the breast specialty clinic of our urban, tertiary care academic medical center between the years 2004-2014. The University of Washington Institutional Review Board reviewed and approved the study (human subjects application with no: \#49769). Clinical, radiological, microbiological, and histological data, as well as management and treatment outcomes were extracted from patient charts. Diagnoses of GM were histopathologically confirmed from deep-tissue samples from either core or excisional biopsies. If obtained at our institution, deeptissue samples were examined with special stains for bacterial, acid-fast, and fungal organisms, and PCR was performed for tuberculous and non-tuberculous mycobacteria. As our diagnostic algorithm evolved, species-level identification of Corynebacterium spp. isolated in culture or identified by PCR were requested from deep-tissue samples and from fluid obtained from incision \& drainage (I\&D) and fine needle aspiration (FNA).

Clinical resolution of GM was defined as a) absence of subjective symptoms such as pain or tenderness and b) lack of evidence of active disease by either clinical breast exam or breast ultrasound. Redevelopment of either symptoms or clinical abnormalities within two months of cessation of therapy was considered an incompletely treated primary episode of GM. Recurrence was defined as redevelopment of symptoms or clinical abnormalities greater than two months after cessation of therapy. 
Data from a chart review was entered and stored in Research Electronic Data Capture (REDCap), a secure web-based software platform (30). Ten percent of the data was spot checked by an independent party. Descriptive statistics were used to describe the demographic and clinical characteristics of the sample. Time to resolution was analyzed using methods for survival time analysis for censored data. Kaplan-Meier curves were used to describe the distribution, and the log rank test was used to test whether time to resolution differed according to patient characteristics.

Forty-two patients with GM were identified by chart review. Six patients had active GM at the time of their last clinic visit and were subsequently lost to follow-up. Their clinical outcome is unknown. The remaining 36 patients were followed up at our clinic to clinical resolution. Patients lost to follow-up were not included in the survival time analysis, Kaplan-Meier curves, or log rank test.

\section{Results}

\section{Demographics}

All patients were women with an average age at disease onset of 32 (range: 18-64) (Table 1). Fifty percent identified as Hispanic, with nearly $1 / 3$ originating from Mexico. The majority of patients

Table 1. Demographics $(n=42)$

\section{Gender}

Female

$100 \%$

Male

$0 \%$

Mean age at onset (years)

$32(18-64)$

Race/ethnicity

Hispanic

Non-Hispanic white

$21 \%$

Asian

Black

Mixed

Country of birth

USA

Mexico

Korea

Vietnam

Honduras

El Salvador

Guatemala

Moldova

Unstated

$12 \%$

Parous

Pregnant at onset

$19 \%$

Breastfeeding at onset

$0 \%$
(93\%) were parous. Eight patients were pregnant at symptom onset.

The most common initial breast symptoms were presence of a mass/ lump, pain/tenderness, and erythema (Table 2). Close to one-quarter of patients had a fever, and $19 \%$ reported erythema nodosum. Patients most commonly presented first to primary care, obstetrics/gynecology, and emergency medicine.

The average duration of symptoms before presentation to our institution's breast clinic was 7.2 months (range: 0.2-32 months). Eighty-one percent of patients received antibiotics prior to their first breast clinic visit and 33\% had been treated with immunosuppressive therapy. Most patients were unable to recall the details regarding those treatments, such as medication names, dosages, and duration.

\section{Radiographic and histologic findings}

Breast ultrasound was performed on 40 (95.2\%) patients, with common findings being a lobulated or irregular hypoechoic mass, sinus tract, and complex fluid collection. A mammogram was obtained in $25(59.5 \%)$ patients and most commonly demonstrated focal

Table 2. Clinical characteristics and presentation ( $n=42)$

Breast symptoms at disease onset

n (\%)

Mass/lump

$37(88.1)$

Pain/tenderness

$34(81.0)$

Erythema

$26(61.9)$

Abscess

$7(16.7)$

Induration

Itching

Fistula

$3(7.1)$

Associated symptoms at disease onset

Fever

$10(23.8)$

Erythema nodosum

Chills

Breast trauma prior to disease onset

Initial presenting specialty

Primary care

$17(40.4)$

Ob/gyn

$8(19.0)$

Emergency medicine/urgent care

Not stated

10 (23.8)

Mean time from symptom onset to breast clinic provider (months)

Mean size of lesion by exam at first breast clinic visit $(\mathrm{cm})$

$7.4 \times 6.6$

Patients who had been treated with antibiotics prior to first breast clinic visit

$34(81.0)$

Patients who had been treated with immunosuppressive therapy prior to first breast clinic visit

$14(33.3)$

Average number of procedures per patient prior to first breast clinic visit
1.8 
asymmetric density, a lobulated irregular mass, and skin thickening. Three patients had a breast magnetic resonance imaging.

Tissue for histopathology was obtained by core needle biopsy in 38 (90.5\%) patients and excisional biopsy in seven (16.7\%) patients. Histology was characterized by lobular-centric active granulomatous inflammation with or without concomitant chronic inflammation and no evidence of malignancy. Associated abscess formation, fat necrosis, and stromal fibrosis were also seen.

\section{Microbiology}

Bacteria were identified in 34 samples from 22/42 patients $(52.3 \%$ ) (Table 3). Diphtheroids (presumptive Corynebacterium spp.) were most commonly identified, followed by Corynebacterium spp. and Propionibacterium acnes (now Cutibacterium acnes).

Twenty-eight patients (66.7\%) presented to their first breast clinic visit with microbiological data obtained from an outside institution. A total of 51 samples were collected from these patients. Twelve patients overall had samples that grew one or more organisms, and nine of these patients had samples that grew presumed Corynebacterium speciated and non-speciated organisms. Fourteen patients either did not have microbiological data prior to their first visit or did have microbiology studies but the results were unclear or unknown. Details regarding antibiotic status when samples were collected was also unknown.

Table 3. Organisms identified from samples both outside and within our institution

Presumed Corynebacterium speciated and non-speciated

\begin{tabular}{|c|c|c|c|c|}
\hline & Organism & No of samples* & Sampling technique & Microbiology test \\
\hline \multirow{3}{*}{$\begin{array}{l}\text { Obtained outside } \\
\text { institution }\end{array}$} & Diphtheroids & 11 & $\begin{array}{c}\text { FNA (4) } \\
\text { I\&D (5) } \\
\text { Biopsy (1) } \\
\text { Superficial sample }{ }^{\dagger}(1)\end{array}$ & Bacterial culture \\
\hline & C. kroppenstedtii & 3 & $\begin{array}{l}\text { FNA (2) } \\
\text { I\&D (1) }\end{array}$ & $\begin{array}{l}\text { Bacterial culture (2) } \\
\text { DNA sequencing (1) }\end{array}$ \\
\hline & $\begin{array}{c}\text { Corynebacterium spp. } \\
\text { (species not determined) }\end{array}$ & 1 & I\&D & Bacterial culture \\
\hline \multirow{2}{*}{$\begin{array}{l}\text { Obtained within } \\
\text { institution }\end{array}$} & C. kroppenstedtii & 2 & $\begin{array}{c}\text { Superficial sample† (1) } \\
\text { FNA (1) }\end{array}$ & Bacterial culture \\
\hline & $\begin{array}{c}\text { Corynebacterium } \\
\text { tuberculostearicum or } \\
\text { Corynebacterium group G2 }\end{array}$ & 1 & Biopsy & Bacterial PCR \\
\hline Other organisms & Organism & No of samples* & Sampling technique & Microbiology test \\
\hline \multirow{4}{*}{$\begin{array}{l}\text { Obtained outside } \\
\text { institution }\end{array}$} & \multirow{3}{*}{ P. acnes } & \multirow{3}{*}{3} & Biopsy (1) & \\
\hline & & & I\&D (1) & Culture \\
\hline & & & Superficial sample ${ }^{\dagger}$ (1) & \\
\hline & $\begin{array}{l}\text { Stenotrophomonas } \\
\text { maltophilia }\end{array}$ & 1 & Biopsy (1) & Culture \\
\hline \multirow{9}{*}{$\begin{array}{l}\text { Obtained within } \\
\text { institution }\end{array}$} & Actinomyces odontolyticus & 1 & Superficial sample ${ }^{\dagger}(1)$ & Culture \\
\hline & $\begin{array}{l}\text { Coagulase negative } \\
\text { staphylococcus }\end{array}$ & 3 & $\begin{array}{c}\text { FNA (1) } \\
\text { Superficial sample }{ }^{\dagger}(2)\end{array}$ & Culture \\
\hline & Micrococcus & 2 & $\begin{array}{l}\text { I\&D (1) } \\
\text { FNA (1) }\end{array}$ & Culture \\
\hline & P. acnes & 1 & Biopsy (1) & Culture \\
\hline & Citrobacter freundii & 1 & I\&D & Culture \\
\hline & Enterococcus & 1 & $I \& D(1)$ & Culture \\
\hline & Klebsiella pneumoniae & 1 & Superficial sample ${ }^{\dagger}(1)$ & Culture \\
\hline & Mycobacterium chelonae & 1 & Biopsy & AFB PCR \\
\hline & Serratia marcescens & 1 & Superficial sample† (1) & Culture \\
\hline
\end{tabular}

* Of 90 samples obtained within and outside our institution, 34 samples from 22/42 patients (52.3\%) grew one or more organisms; †Superficial samples were obtained from either nipple discharge or fluid from draining sinus tracts 
Within our institution, a total of 39 microbiology samples were obtained from 24 patients (57.1\%). 21/39 (53.8\%) samples were collected while the patient was currently on antibiotics or had finished a course of antibiotics within the last week. Ten patients overall had samples that grew one or more organisms.

\section{Management}

Six patients (14\%) had active GM at the time of their last visit and were subsequently lost to follow-up. The remaining 36 were followed to clinical resolution. Their management is outlined in Table 4.

Antibiotics were our preferred first-line medical therapy and were used in 33/36 (91.7\%) patients. The mean duration of antibiotic therapy was $7.0 \pm 4.5$ months. Clarithromycin was our antibiotic of choice given its lipophilic properties and its ability to concentrate within macrophage-derived elements of granulomas. It was the initial agent used in 15 of the 33 patients $(45.5 \%)$ treated with antibiotics. Patients were treated with typical doses of clarithromycin at $500 \mathrm{mg}$ twice daily. The remaining 18 patients received an alternate antibiotic because they either a) were already on an alternate antibiotic at the time of their first breast clinic visit and were showing clinical improvement on this agent or b) had a contraindication to clarithromycin, such as pregnancy or allergy. Twelve of these patients were eventually switched to clarithromycin due to lack of continued clinical improvement on an alternate antibiotic. There were no major adverse effects of clarithromycin therapy. Two patients experienced gastrointestinal side effects, and one patient had QTC prolongation and was therefore transitioned to high-dose amoxicillin.

Eleven patients required adjunctive therapy with prednisone due to worsening or plateau of symptoms on antibiotic therapy. Steroid doses varied, but we generally avoided high-dose steroid therapy and typically started at $20-30 \mathrm{mg} /$ day followed by taper based on clinical improvement. The mean duration of steroid therapy was $4.3 \pm 2.5$ months. No patients required discontinuation of steroid therapy due to adverse effects, although two patients declined initiating treatment with steroids due to concerns about weight gain.

Surgery for therapeutic, not diagnostic, purposes included incision and drainage in seven patients, FNA in eight patients, and excision of fistulous tract in one patient. No patients had large-volume excisions.

Table 4. Management of patients followed to clinical resolution $(n=36)$

Therapy

Antibiotics only*

Antibiotics + steroids*

Antibiotics + surgery*

Antibiotics + steroids + surgery*

Steroids only

Observation only

* $81.8 \%$ of patients treated with antibiotics received a course of macrolides

\section{Outcomes}

The average time from the first breast clinic visit to clinical resolution was $8.0 \pm 4.6$ months. The estimated probability of resolution was $41 \%$ within 6 months, 37\% between 6 and 12 months, and 22\% in more than 12 months. Time to resolution was not significantly associated with baseline lesion size ( $p=0.135)$, identification of a pathogenic organism $(p=0.738)$, or time since symptom onset $(p=0.127)$.

Recurrence was defined as redevelopment of symptoms or clinical abnormalities greater than two months after cessation of therapy. In all, $17 / 36(47 \%)$ patients were followed by either a breast clinic provider or another provider within our system after clinical resolution. Five of these patients (29\%) developed recurrence of GM. The average number of months from clinical resolution to recurrence was 30.2.

\section{Discussion and Conclusion}

The results of our study lend support to the theory that an infectious process, particularly one initiated by corynebacteria, may play a role in the pathogenicity of GM. Historically, corynebacteria have been overlooked due to a lack of association of these bacteria with infection. However, the hypothesis that these organisms can be pathogenic in the breast is gaining momentum (20-23), and is consistent with what is understood about granuloma formation in other settings. Mycobacterium tuberculosis, for example, is also found in lipid-laden macrophages and produces a chronic inflammatory response, requiring long courses of antibiotics for effective treatment.

It is worth noting that $12 / 28$ patients who presented to our breast clinic with microbiology data obtained from an outside institution had a microorganism identified in one or more samples. Though this may seem like a high yield compared with the low rates of positive cultures seen in prior studies, the majority of organisms identified in these patients were diphtheroids. For a number of reasons, diphtheroids may have been under-reported or under-detected in prior studies, including a paucity of organisms present in clinical specimens, fastidious growth requirements of C. kroppenstedii, and dismissal of diphtheroids isolated in culture as contaminants. Additionally, details about how tissue samples were obtained and cultured are not included in many studies. If samples were of superficial rather than deep-tissue, this may also explain the lower yield observed in other papers. Furthermore, antibiotic status at time of sampling can also affect the culture results. This could explain the relatively lower yield from cultures obtained at our institution, as a high percentage of patients were on antibiotics when samples were obtained for culture.

Despite the increasing literature suggesting a pathogenic role of Corynebacterium spp. in inflammatory breast disease and GM, it is not commonplace to request that laboratory staff consider isolation and identification of corynebacteria in specimens sent for analysis. We therefore suggest that when sending samples to the laboratory, clinicians alert laboratory staff to possible bacterial etiology and request that they provide a species-level identification of any corynebacteria recovered in culture. To evaluate for other secondary infectious etiologies of GM and improve overall yield, we suggest that samples (tissue, fluid, or purulent material) be submitted for Gram staining, bacterial culture, fungal staining and culture, and acid-fast bacilli staining and culture. To improve recovery of $C$. kroppenstedtii in particular, laboratories 
should consider adding Tween 80 (a lipid source) and esculin to growth media. Cultures should be incubated for a sufficient duration to recover slow-growing organisms such as C. kroppenstedtii. As the culture can be negative, the addition of a broad range bacterial, fungal, or acid-fast bacillus PCR can be considered to improve detection of infectious etiologies.

Based on the supposition that GM is the result of a bacterial infection, we prioritized treatment with prolonged courses of lipophilic antibiotics for adequate tissue penetrance within lipid-filled spaces. It is difficult to compare the results presented here to other studies in terms of time to resolution, as many existing studies do not include information about when a case was considered to have started ( $1^{\text {st }}$ presentation vs $1^{\text {st }}$ clinical encounter with subspecialist or date of diagnosis). One recent systematic review of cases from around the world demonstrated an average delay in diagnosis of four to five months (31). Still, accounting for heterogeneity in reporting of "start" and "completion" of therapy, our treatment strategy appears to be similar to those of other studies in terms of time to resolution, with a mean antibiotic therapy duration of seven months, and a mean total time from "first visit" to "resolution" of eight months.

While additional studies are needed to further evaluate the emerging theory regarding the role of corynebacteria in GM, given the growing evidence and the low morbidity associated with antibiotic treatment, it is reasonable for clinicians to consider treating GM with empiric antibiotic therapy in cases where an organism is not identified. Should a clinician choose this strategy, we suggest treatment with either clarithromycin $500 \mathrm{mg}$ twice daily or doxycycline $100 \mathrm{mg}$ twice daily. Clarithromycin is preferred in the absence of contraindications to its use. Like other macrolides, it concentrates in macrophages, which are integral to the formation and structure of granulomas (32). Possible side effects of clarithromycin include gastrointestinal side effects, elevated liver function tests, and QT prolongation. Water soluble agents used at the higher end of dosing (e.g., Amoxicillin $1000 \mathrm{mg}$ every 8 hours) are another option if the above are contraindicated or not tolerated.

The response to antibiotic treatment can be slow, and careful measurement of the area of induration from visit to visit is essential. Based on our experience, signs of improvement-reduction in size, reduction in pain and erythema, organization of the phlegmon-like involvement into a drainable abscess — should be evident within 2-4 weeks. Clinic visits should be scheduled every 1-3 months to allow close monitoring for the above signs. Worsening or the absence of improvement should prompt a change to a second antibiotic. Antibiotic therapy should be continued until clinical resolution. If disease fails to improve with antibiotic therapy, addition of prednisone at $20-30 \mathrm{mg}$ a day for 10-14 days with tapering based on the clinical response can be added to the antibiotic. Clinicians should be aware of the multitude of possible side effects of steroid therapy, including weight gain, mood disorders, hyperglycemia, hypertension, immunosuppression, and osteoporosis. The risk of these side effects can be mitigated by limiting the dose and duration of steroids to the minimum necessary for clinical effectiveness.

In our patients, we opted to avoid treatment with surgical excision, aside from excision of the fistulous tract in one patient. Multiple a longer course to recovery than surgical excision, patients may in fact achieve resolution with non-surgical treatments and thus potentially be spared disfiguring surgeries or surgical complications $(5,7,11,33)$. Conservative needle drainage of well-formed abscesses and surgical drainage of larger ones did play a role in our treatment strategy but was reserved for refractory cases or for patients who desired more rapid symptom relief.

Confirmation of the superiority of an antibiotic-centered approach versus surgery, immunosuppressive treatment, or observation only requires a randomized trial. The roles of multi-drug therapy and intralesional injections are largely unexplored.

This study had a predominance of non-White patients, the majority being Hispanic. This pattern has been echoed in other studies $(6,8$, 34) in the United States and is of unclear significance. Additionally, the Mediterranean region, specifically Turkey, has made a large contribution to the body of literature about GM, which raises the question of whether this disease has an epidemiologic clustering in certain ethnicities (35-37). One hypothesis to explain this may be due to differing natal microbiomes or environmental exposures in different ethnic groups. More research is needed to understand the epidemiology of this disease.

This study is limited in its scope to one academic center, which may limit its generalizability. However, our institution is typically a referral center for this disease, seeing perhaps a much larger volume than would be predicted based on the prevalence of the disease in the general population. Additional limitations include the retrospective nature of the data collection and limitations on access to patients' microbiological and medication details from outside institutions.

In summary, this study suggests that GM may be the result of a primarily infectious process and highlights the need for further research. Until there is more definitive data in this area, we suggest that clinicians request inclusion of Corynebacterium spp. in the evaluation of samples sent for laboratory analysis. Extended courses of lipophilic antibiotics such as clarithromycin may be a viable treatment option; this approach has the potential to reduce the application of more harmful interventions such as prolonged steroid use (mean duration of steroid use only four months) or surgery, including mastectomy.

\section{Acknowledgements}

We would like to thank Nina Tan, MD for help with early literature review.

Ethics Committee Approval: The University of Washington Institutional Review Board reviewed and approved the study on 6/2/2015 (human subjects application with no: \#49769).

Informed Consent: Granted waivers of consent by the University of Washington Institutional Review Board in Subcommittee EB.

Peer-review: Externally peer-reviewed.

Conflict of Interest: No conflict of interest was declared by the authors.

Financial Disclosure: The authors declared that this study received no financial support. 


\section{Authorship Contributions}

Conception: A.H.M., M.B.L.; Design: A.H.M., M.B.L.; Supervision: M.B.L.; Editing: A.H.M.; Data Collection and/or Processing: M.S.W., M.B.L.; Analysis and/or Interpretation: M.S.W., M.B.L.; Literature Review: M.S.W., M.B.L., L.B.; Writing: M.S.W., M.B.L., L.B.; Critical Review: M.B.L.

\section{References}

1. Kessler E, Wolloch Y. Granulomatous mastitis: a lesion clinically simulating carcinoma. Am J Clin Pathol 1972; 58: 642-646. (PMID: 4674439) [Crossref]

2. Sheybani F, Sarvghad M, Naderi HR, Gharib M. Treatment for and clinical characteristics of granulomatous mastitis. Obstet Gynecol 2015; 125: 801-807. (PMID: 25751209) [Crossref]

3. Pereira FA, Mudgil AV, Macias ES, Karsif K. Idiopathic granulomatous lobular mastitis. Int J Dermatol 2012; 51: 142-151. (PMID: 22250621) [Crossref]

4. Patel RA, Strickland P, Sankara IR, Pinkston G, Many W Jr, Rodriguez M. Idiopathic granulomatous mastitis: case reports and review of literature. J Gen Intern Med 2010; 25: 270-273. (PMID: 20013067) [Crossref]

5. Bouton ME, Jayaram L, O'Neill PJ, Hsu CH, Komenaka IK. Management of idiopathic granulomatous mastitis with observation. Am J Surg 2015; 210: 258-262. (PMID: 25746911) [Crossref]

6. Davis J, Cocco D, Matz S, Hsu CH, Brown MJ, Lee J, et al. Re-evaluating if observation continues to be the best management of idiopathic granulomatous mastitis. Surgery 2019; 166: 1176-1180. (PMID: 31400951) [Crossref]

7. Joseph KA, Luu X, Mor A. Granulomatous mastitis: a New York public hospital experience. Ann Surg Oncol 2014; 21: 4159-4163. (PMID: 25008030) [Crossref]

8. Pandey TS, Mackinnon JC, Bressler L, Millar A, Marcus EE, Ganschow PS. Idiopathic granulomatous mastitis--a prospective study of 49 women and treatment outcomes with steroid therapy. Breast J 2014; 20: 258-266. (PMID: 24673796) [Crossref]

9. Sakurai K, Fujisaki S, Enomoto K, Amano S, Sugitani M. Evaluation of follow-up strategies for corticosteroid therapy of idiopathic granulomatous mastitis. Surg Today 2011; 41: 333-337. (PMID: 21365412) [Crossref]

10. Freeman CM, Xia BT, Wilson GC, Lewis JD, Khan S, Lee SJ, et al. Idiopathic granulomatous mastitis: a diagnostic and therapeutic challenge. Am J Surg 2017; 214: 701-706. (PMID: 28739122) [Crossref]

11. Akbulut S, Arikanoglu Z, Senol A, Sogutcu N, Basbug M, Yeniaras E, et al. Is methotrexate an acceptable treatment in the management of idiopathic granulomatous mastitis? Arch Gynecol Obstet 2011; 284: 1189-1195. (PMID: 21207047) [Crossref]

12. Valeyre D, Prasse A, Nunes H, Uzunhan Y, Brillet PY, Müller-Quernheim J. Sarcoidosis. Lancet 2014; 383: 1155-1167. (PMID: 24090799) [Crossref]

13. Li J. Diagnosis and treatment of 75 patients with idiopathic lobular granulomatous mastitis. J Invest Surg 2019; 32: 414-420. (PMID: 29381437) [Crossref]

14. Prasad S, Jaiprakash P, Dave A, Pai D. Idiopathic granulomatous mastitis: an institutional experience. Turk J Surg 2017; 33: 100-103. (PMID: 28740959) [Crossref]

15. Akcan A, Oz AB, Dogan S, Akgun H, Akyuz M, Ok E, et al. Idiopathic granulomatous mastitis: comparison of wide local excision with or without corticosteroid therapy. Breast Care (Basel) 2014; 9: 111-115. (PMID: 24944554) [Crossref]

16. Asoglu O, Ozmen V, Karanlik H, Tunaci M, Cabioglu N, Igci A, et al. Feasibility of surgical management in patients with granulomatous mastitis. Breast J 2005; 11: 108-114. (PMID: 15730456) [Crossref]
17. Bani-Hani KE, Yaghan RJ, Matalka I, Shatnawi NJ. Idiopathic granulomatous mastitis: time to avoid unnecessary mastectomies. Breast J 2004; 10: 318-322. (PMID: 15239790) [Crossref]

18. Chirappapha P, Thaweepworadej P, Supsamutchai C, Biadul N, Lertsithichai P. Idiopathic granulomatous mastitis: a retrospective cohort study between 44 patients with different treatment modalities. Ann Med Surg (Lond) 2018; 36: 162-167. (PMID: 30479764) [Crossref]

19. Wilson JP, Massoll N, Marshall J, Foss RM, Copeland EM, Grobmyer SR. Idiopathic granulomatous mastitis: in search of a therapeutic paradigm. Am Surg 2007; 73: 798-802. (PMID: 17879688) [Crossref]

20. Taylor GB, Paviour SD, Musaad S, Jones WO, Holland DJ. A clinicopathological review of 34 cases of inflammatory breast disease showing an association between corynebacteria infection and granulomatous mastitis. Pathology 2003; 35: 109-119. (PMID: 12745457) [Crossref]

21. Paviour S, Musaad S, Roberts S, Taylor G, Taylor S, Shore K, et al. Corynebacterium species isolated from patients with mastitis. Clin Infect Dis 2002; 35: 1434-1440. (PMID: 12439810) [Crossref]

22. Wong SCY, Poon RWS, Chen JHK, Tse H, Lo JYC, Ng TK, et al. Corynebacterium kroppenstedtii Is an emerging cause of mastitis especially in patients with psychiatric illness on antipsychotic medication. Open Forum Infect Dis 2017; 4: ofx096. doi: 10.1093/ofid/ofx096 (PMID: 28852671) [Crossref]

23. Dobinson HC, Anderson TP, Chambers ST, Doogue MP, Seaward L, Werno AM. Antimicrobial treatment options for granulomatous mastitis caused by corynebacterium species. J Clin Microbiol 2015; 53: 28952899. (PMID: 26135858) [Crossref]

24. Kutsuna S, Mezaki K, Nagamatsu M, Kunimatsu J, Yamamoto $\mathrm{K}$, Fujiya $\mathrm{Y}$, et al. Two cases of granulomatous mastitis caused by corynebacterium kroppenstedtii infection in nulliparous young women with hyperprolactinemia. Intern Med 2015; 54: 1815-1818. (PMID: 26179543) [Crossref]

25. Tauch A, Schneider J, Szczepanowski R, Tilker A, Viehoever P, Gartemann $\mathrm{KH}$, et al. Ultrafast pyrosequencing of corynebacterium kroppenstedtii DSM44385 revealed insights into the physiology of a lipophilic corynebacterium that lacks mycolic acids. J Biotechnol 2008; 136: 22-30. (PMID: 18430482) [Crossref]

26. Goh Z, Tan AL, Madhukhumar P, Yong WS. Recurrent corynebacterium kroppenstedtii breast abscess in a young asian female. Breast J 2015; 21 : 431-432. (PMID: 26136183) [Crossref]

27. Gibson SER, Harrison J, Cox JAG. Modelling a silent epidemic: a review of the in vitro models of latent tuberculosis. Pathogens 2018; 7: 88. (PMID: 30445695) [Crossref]

28. Silva LC, Geluk A, Arnone M, Romiti R, Franken KC, Duarte AJ, et al. Infliximab partially impairs the anti-Mycobacterium tuberculosis immune responses of severe psoriasis patients with positive tuberculin skin-test. J Eur Acad Dermatol Venereol 2012; 26: 319-324. (PMID: 21623925) [Crossref]

29. Johnson MG, Leal S, Plongla R, Leone PA, Gilligan PH. The brief case: recurrent granulomatous mastitis due to corynebacterium kroppenstedtii. J Clin Microbiol 2016; 54: 1938-1941. (PMID: 27458268) [Crossref]

30. Harris PA, Taylor R, Thielke R, Payne J, Gonzalez N, Conde JG. Research electronic data capture (REDCap)--a metadata-driven methodology and workflow process for providing translational research informatics support. J Biomed Inform 2009; 42: 377-381. (PMID: 18929686) [Crossref]

31. Martinez-Ramos D, Simon-Monterde L, Suelves-Piqueres C, QueraltMartin R, Granel-Villach L, Laguna-Sastre JM, et al. Idiopathic granulomatous mastitis: A systematic review of 3060 patients. Breast J 2019; 25: 1245-1250. (PMID: 31273861) [Crossref]

32. Jain R, Danziger LH. The macrolide antibiotics: a pharmacokinetic and pharmacodynamic overview. Curr Pharm Des 2004; 10: 3045-3053. (PMID: 31273861) [Crossref] 
33. Lai EC, Chan WC, Ma TK, Tang AP, Poon CS, Leong HT. The role of conservative treatment in idiopathic granulomatous mastitis. Breast $\mathrm{J}$ 2005; 11: 454-456. (PMID: 16297091) [Crossref]

34. Idiopathic Granulomatous Mastitis in Hispanic Women --- Indiana, 2006--2008. Last Accessed Date : 12.02.2009 Available from: https:// www.cdc.gov/mmwr/preview/mmwrhtml $/ \mathrm{mm} 5847 \mathrm{a} 1 . \mathrm{htm}$. [Crossref]

35. Karanlik H, Ozgur I, Simsek S, Fathalizadeh A, Tukenmez M, Sahin D, et al. Can steroids plus surgery become a first-line treatment of idiopathic granulomatous mastitis? Breast Care (Basel) 2014; 9: 338-342. (PMID: 25759614) [Crossref]
36. Korkut E, Akcay MN, Karadeniz E, Subasi ID, Gursan N. Granulomatous mastitis: a ten-year experience at a University Hospital. Eurasian J Med 2015; 47: 165-173. (PMID: 26644764) [Crossref]

37. Oran ES, Gurdal SO, Yankol Y, Oznur M, Calay Z, Tunaci M, et al. Management of idiopathic granulomatous mastitis diagnosed by core biopsy: a retrospective multicenter study. Breast J 2013; 19: 411-418. (PMID: 23663101) [Crossref] 